\title{
The gamma-Weibull distribution revisited
}

\author{
TIBOR K. POGÁNY ${ }^{1}$ and RAM K. SAXENA ${ }^{2}$ \\ ${ }^{1}$ Faculty of Maritime Studies, University of Rijeka, HR-51000 Rijeka, Studentska 2, Croatia \\ ${ }^{2}$ Department of Mathematics and Statistics, Faculty of Science, Jai Narain Vyas University, Jodhpur-342004, India
}

Manuscript received on June 25, 2008; accepted for publication on August 3, 2009

\begin{abstract}
The five parameter gamma-Weibull distribution has been introduced by Leipnik and Pearce (2004). Nadarajah and Kotz (2007) have simplified it into four parameter form, using hypergeometric functions in some special cases. We show that the probability distribution function, all moments of positive order and the characteristic function of gamma-Weibull distribution of a random variable can be explicitely expressed in terms of the incomplete confluent Fox-Wright Psi-function, which is recently introduced by Srivastava and Pogány (2007). In the same time, we generalize certain results by Nadarajah and Kotz that follow as special cases of our findings.
\end{abstract}

Key words: gamma distribution, Weibull distribution, confluent Fox-Wright ${ }_{1} \Psi_{0}$, incomplete confluent Fox-Wright ${ }_{1} \psi_{0}$.

\section{INTRODUCTION AND RESULTS REQUIRED}

Multiplying and then renormalizing the product of densities of gamma and Weibull distributions (Leipnik and Pearce 2004) defined the so-called gamma-Weibull distribution. The related probability density function (pdf in the sequel) was simplified by Nadarajah and Kotz (2007, Eq. (1.1)) in the form

$$
f_{g W}(x)=K x^{\alpha-1} e^{-\mu x-a x^{r}} \chi_{(0, \infty)}(x) \quad(\theta:=(\alpha, \mu, a, r)>0),
$$

where $\chi_{A}(x)$ denotes the indicator of $\{x \in A\}$, while $K=K(\alpha, \mu, a, r)$ is the normalizing constant. In what follows we write $\xi \sim g W(\theta)$ when the r.v. $\xi$ has the gamma-Weibull distribution with parameter $\theta$.

Leipnik and Pearce obtained the characteristic function and the moments of gamma-Weibull distribution. Nadarajah and Kotz simplified these results in a modestly simpler form using finite sums of generalized hypergeometric function ${ }_{p+1} F_{q}$ (Nadarajah and Kotz 2007, Eqs. (2.1-2)), when $r=p / q ; p, q \geq 1$ are co-prime integers.

2010 Mathematics subject classification: Primary 33C90; Secondary 62E99.

Correspondence to: Tibor K. Pogány

E-mail: poganj@pfri.hr 
We will show here that the cumulative distribution function (CDF), the moments of all orders having positive real parts and the characteristic function (chf) can be represented explicitely in terms of the socalled confluent Fox-Wright function ${ }_{1} \Psi_{0}$ and its incomplete variant form ${ }_{1} \psi_{0}$ for all $r>0$, thereby simplifying and generalizing mainly the results (Nadarajah and Kotz 2007). The incomplete ${ }_{p} \psi_{q}$ is studied very recently by Srivastava and Pogány (2007). In what follows, the symbol ${ }_{p} \Psi_{q}$ stands for the Fox-Wright generalization of the hypergeometric function ${ }_{p} \digamma_{q}$, with $p$ numerator and $q$ denominator parameters, defined by

$$
\begin{aligned}
{ }_{p} \Psi_{q}\left[\begin{array}{c}
\left(\alpha_{1}, A_{1}\right), \cdots,\left(\alpha_{p}, A_{p}\right) \\
\left(\beta_{1}, B_{1}\right), \cdots,\left(\beta_{q}, B_{q}\right)
\end{array} \mid x\right] \\
={ }_{p} \Psi_{q}\left[\begin{array}{c}
\left(\alpha_{p}, A_{p}\right) \\
\left(\beta_{q}, B_{q}\right)
\end{array} \mid x\right]:=\sum_{m=0}^{\infty} \frac{\prod_{\ell=1}^{p} \Gamma\left(\alpha_{\ell}+A_{\ell} m\right)}{\prod_{\ell=1}^{q} \Gamma\left(\beta_{\ell}+B_{\ell} m\right)} \frac{x^{m}}{m !} \\
\\
\quad\left(A_{\ell}, B_{j} \in \mathbb{R}_{+}, \ell=\overline{1, p}, j=\overline{1, q} ; \quad 1+\sum_{\ell=1}^{q} B_{\ell}-\sum_{j=1}^{p} A_{j}>0\right),
\end{aligned}
$$

for suitably bounded values of $|x|$ and in terms of Euler's Gamma function

$$
\Gamma(z)=\int_{0}^{\infty} x^{z-1} e^{-z} \mathrm{~d} z \quad(\Re\{z\}>0),
$$

where an empty product in (2) is to be interpreted (as usual) to be 1 (Mathai and Saxena 1978), (Srivastava et al. 1982). In terms of the analytically continued Gamma-function $\left.\Gamma(z), z \in \mathbb{C} \backslash \mathbb{Z}_{-}^{0}:=\{0,-1,-2, \cdots\}\right)$, the incomplete Gamma-function $\gamma(z, a)$ (Gradshteyn and Ryzhik 2000, 8.350 1.) is given by truncating the integration domain of Gamma-function to $[0, \omega]$, i.e.

$$
\gamma(z, \omega):=\int_{0}^{\omega} x^{z-1} e^{-x} \mathrm{~d} x \quad(\Re\{z\}>0, \omega \geq 0) .
$$

Following (Srivastava and Pogány 2007, Eq. (6)), we define the so-called incomplete Fox-Wright function ${ }_{p} \psi_{q}$ in the form

$$
\begin{gathered}
{ }_{p} \psi_{q}\left[\left.\begin{array}{c}
\left(\alpha_{p}, A_{p}\right) \\
\left(\beta_{q}, B_{q}\right)
\end{array}\right|_{\gamma}(x, \omega)\right]:=\sum_{m=0}^{\infty} \frac{\prod_{\ell=1}^{p} \gamma\left(\alpha_{\ell}+A_{\ell} m, \omega\right)}{\prod_{\ell=1}^{q} \gamma\left(\beta_{\ell}+B_{\ell} m, \omega\right)} \frac{x^{m}}{m !} \\
\mathcal{P}:=\left(A_{\ell}, B_{j} \in \mathbb{R}_{+}, \ell=\overline{1, p}, j=\overline{1, q} ; \quad 1+\sum_{\ell=1}^{q} B_{\ell}-\sum_{j=1}^{p} A_{j}>0 ; \quad \omega \geq 0\right) .
\end{gathered}
$$

It is interesting to remark, that the definition (3) immediately yields the limit relationship

$$
\lim _{\omega \rightarrow \infty} \psi_{q}\left[\left.\begin{array}{c}
\left(\alpha_{p}, A_{p}\right) \\
\left(\beta_{q}, B_{q}\right)
\end{array}\right|_{\gamma}(x, \omega)\right] \equiv{ }_{p} \Psi_{q}\left[\begin{array}{c}
\left(\alpha_{p}, A_{p}\right) \\
\left(\beta_{q}, B_{q}\right)
\end{array} \mid x\right] .
$$

\section{THE GAMMA-WEIBULL $g W(\theta)$ DISTRIBUTION}

Cutting off to $(0, z)$ the integration domain $\mathbb{R}_{+}$of the integral $\mathcal{I}(\alpha, \mu, a, r)$ introduced by Nadarajah and Kotz (2007, Eq. (2.1)), we get the resulting integral

$$
\mathcal{I}_{g W}(\theta \mid \omega):=\int_{0}^{\omega} x^{\alpha-1} \exp \left\{-\mu x-a x^{r}\right\} \mathrm{d} x \quad(\theta>0),
$$


such that it is closely connected to $g W(\theta)$ distribution. We express now $\mathcal{I}_{g W}(\theta \mid \omega)$ in terms of the incomplete confluent Fox-Wright ${ }_{1} \psi_{0}$ for positive real $r \in \mathbb{R}_{+}$extending the related result by Nadarajah and Kotz (2007, Eq. (2.2)), which was obtained for rational positive $r \in \mathbb{Q}_{+}$.

LEMMA 1.

$$
\mathcal{I}_{g W}(\theta \mid \omega)= \begin{cases}\frac{\mu^{-\alpha}{ }_{1} \psi_{0}[(\alpha, r)}{\gamma(\alpha,(\mu+a) \omega)} & 0<r<1 \\ (\mu+a)^{\alpha} & r=1 \\ \frac{1}{r a^{\alpha / r}}{ }_{1} \psi_{0}\left[\left.{ }^{(\alpha / r, 1 / r)}\right|_{\gamma}\left(-\mu a^{-1 / r}, \omega^{r}\right)\right] & r>1\end{cases}
$$

ProOF. Expanding exp $\left\{-a x^{r}\right\}$ into Maclaurin series, interchanging the order of the integration and summation and finally using definition of $\gamma(z ; \omega)$, then we easily confirm that

$$
\mathcal{I}_{g W}(\theta \mid \omega)=\frac{1}{\mu^{\alpha}} \sum_{n=0}^{\infty} \gamma(\alpha+r n, \omega) \frac{\left(-a \mu^{-r}\right)^{n}}{n !} \quad(r \in(0,1)) .
$$

which is the assertion of the Lemma. Since the constraint $\mathcal{P}$ in (3) being now ensured with $r<1$, so the series (7) converges. The case $r=1$ results in the incomplete gamma function.

Finally, when $r>1$, replacing $x \mapsto x^{r}$ in the integral (5), we easily deduce

$$
\mathcal{I}_{g W}(\alpha, \mu, a, r \mid \omega)=r^{-1} \mathcal{I}_{g W}\left(\alpha / r, a, \mu, 1 / r \mid \omega^{r}\right) .
$$

This confirms the assertion of the Lemma.

With the help of (4), we clearly deduce the limiting case

$$
\mathcal{I}_{g W}(\theta):=\lim _{\omega \rightarrow \infty} \mathcal{I}_{g W}(\theta \mid \omega)
$$

and a fortiori the limiting results of Lemma 1 . We, therefore, arrive at

COROLlary 1.1 .

$$
\mathcal{I}_{g W}(\theta)= \begin{cases}\mu^{-\alpha}{ }_{1} \Psi_{0}\left[(\alpha, r) \mid-\frac{a}{\mu^{r}}\right] & 0<r<1 \\ \frac{\Gamma(\alpha)}{(\mu+a)^{\alpha}} & r=1 \\ \frac{1}{r a^{\alpha / r}}{ }_{1} \Psi_{0}\left[(\alpha / r, 1 / r) \mid-\frac{\mu}{a^{1 / r}}\right] & r>1\end{cases}
$$


REMARK 1. The case $r=1$ in (Nadarajah and Kotz 2007, Eq. (2.2)) is erroneous - there should be $\Gamma(\alpha)$ in the numerator.

THEOREM 1. Let the random variable $\xi \sim g W(\theta), \theta>0$ be with pdf (1). Then, the pdf $f_{g W}(x)$ and the PDF $F_{g W}(x)$ of r.v. $\xi$ are of the form:

$$
\begin{aligned}
& f_{g W}(x)=\frac{x^{\alpha-1} \exp \left\{-\mu x-a x^{r}\right\}}{\mathcal{I}_{g W}(\theta)} \chi_{(0, \infty)}(x),
\end{aligned}
$$

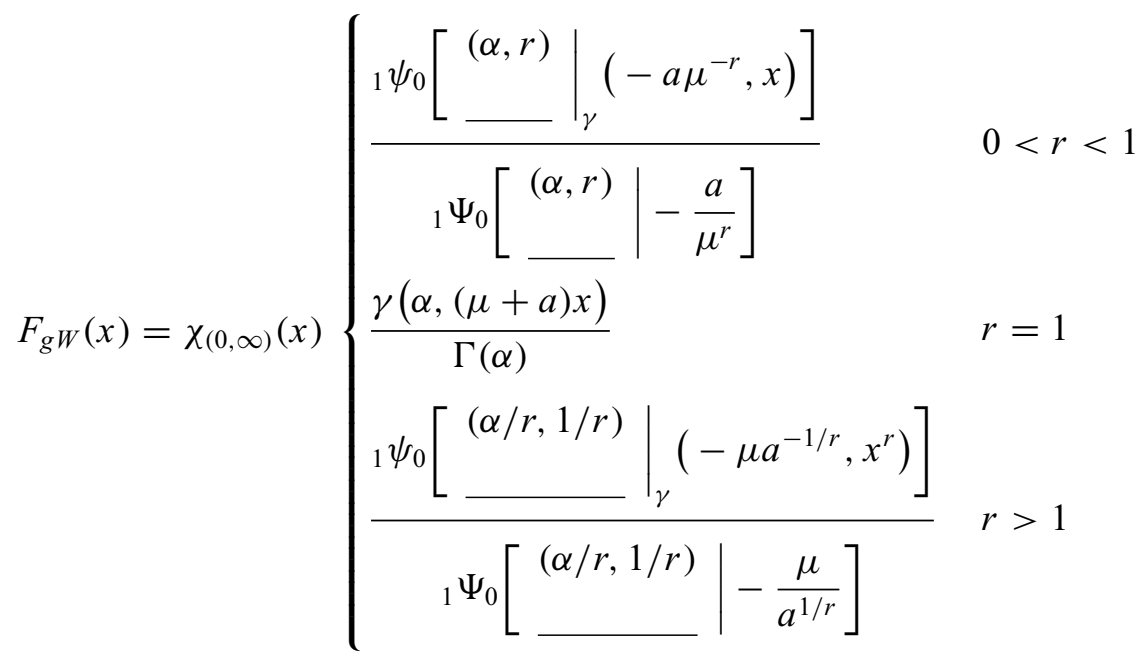

respectively.

Proof. To prove (10), we need the exact value of the normalizing constant $K$, such that appears in (1). But it is straightforward that $K^{-1}=\mathcal{I}_{g W}(\theta)$. As $\mathrm{P}\{\xi \leq 0\}=0$ hance, we deduce that

$$
F_{g W}(x)=\frac{1}{\mathcal{I}_{g W}(\theta)} \int_{0}^{x} t^{\alpha-1} \exp \left\{-\mu t-a t^{r}\right\} \mathrm{d} t=\frac{\mathcal{I}_{g W}(\theta \mid x)}{\mathcal{I}_{g W}(\theta)}
$$

Expanding the last relation into three formulæ with respect to the related domains of $r$, by the Lemma 1 and Corollary 1.1 we conclude (11).

\section{MOMENTS AND CHARACTERISTIC FUNCTION}

We now derive the moments $\mathrm{E} \xi^{\beta}$ and the characteristic function $\phi_{g W}(t)$ of the Gamma-Weibull distribution. All these results are expressed in terms of the confluent Fox-Wright function ${ }_{1} \Psi_{0}$ via the integral $\mathcal{I}_{g W}(\theta)$. 
THEOREM 2. The moment $\mathrm{E} \xi^{\beta}$ of order $\beta$ of the r.v. $\xi \sim g W(\theta)$ is given by

$$
\mathrm{E} \xi^{\beta}= \begin{cases}\frac{{ }_{1} \Psi_{0}\left[(\alpha+\beta, r) \mid-\frac{a}{\mu^{r}}\right]}{\mu^{\beta}{ }_{1} \Psi_{0}\left[(\alpha, r) \mid-\frac{a}{\mu^{r}}\right]} & 0<r<1 \\ \frac{\Gamma(\alpha+\beta)}{\Gamma(\alpha)(\mu+a)^{\beta}} \mid-\frac{\mu}{\left.a^{1 / r}\right]} & r=1 \\ \frac{{ }_{1} \Psi_{0}[(\alpha / r+\beta / r, 1 / r)}{a^{\beta / r}{ }_{1} \Psi_{0}[(\alpha / r, 1 / r)} \mid-\frac{\mu}{\left.a^{1 / r}\right]} & r>1\end{cases}
$$

whenever $\alpha+\Re\{\beta\}>0$.

Moreover, the associated $\mathbf{c h f} \phi_{g W}(t)$ is given by

$$
\phi_{g W}(t)= \begin{cases}\left(\frac{\mu}{\mu-\mathrm{i} t}\right)^{\alpha} \cdot \frac{{ }_{1} \Psi_{0}\left[(\alpha, r) \mid-\frac{a}{(\mu-\mathrm{i} t)^{r}}\right]}{{ }_{1} \Psi_{0}\left[(\alpha, r) \mid-\frac{a}{\mu^{r}}\right]} & 0<r<1 \\ \left(\frac{\mu+a}{\mu+a-\mathrm{i} t}\right)^{\alpha} & r=1 \\ \frac{{ }_{1} \Psi_{0}\left[(\alpha / r, 1 / r) \mid \frac{\mathrm{i} t-\mu}{a^{1 / r}}\right]}{{ }_{1} \Psi_{0}\left[(\alpha / r, 1 / r) \mid-\frac{\mu}{a^{1 / r}}\right]} & r>1\end{cases}
$$

ProOF. Let us take a certain parameter $\beta$, which satisfies $\alpha+\Re\{\beta\}>0$. Then, we have

$$
\mathrm{E} \xi^{\beta}=K \int_{0}^{\infty} x^{\beta} f_{g W}(x) \mathrm{d} x=K \mathcal{I}_{g W}(\alpha+\beta, \mu, a, r) .
$$

Finally, for the chf, we have

$$
\phi_{g W}(t)=\mathrm{E}\left(e^{\mathrm{i} t \xi}\right)=K \int_{0}^{\infty} e^{\mathrm{i} t x} f_{g W}(x) \mathrm{d} x=K \mathcal{I}_{g W}(\alpha, \mu-\mathrm{i} t, a, r) .
$$

Now, replacing $\mu-\mathrm{i} t \mapsto \mu$ in $\mathcal{I}_{g W}(\theta)$, we immediately obtain (13). 


\section{CONCLUDING REMARKS}

A. The confluent Fox-Wright functions ${ }_{1} \Psi_{0},{ }_{1} \psi_{0}$ are defined by series containing gamma function and incomplete gamma function assuming $r \in(0,1)$, we have

$$
{ }_{1} \psi_{0}\left[(\alpha, r)||_{\gamma}(x, \omega)\right]=\sum_{n=0}^{\infty} \frac{\gamma(\alpha+r n, \omega)}{n !} x^{n}=\omega^{\alpha} \sum_{n=0}^{\infty}{ }_{1} \digamma_{1}\left[\begin{array}{c}
\alpha+r n \\
\alpha+r n+1
\end{array} \mid \omega\right] \frac{\left(\omega^{r} x\right)^{n}}{(\alpha+r n) n !},
$$

because of (Srivastava et al. 1982, Eq. A25, p. 244)

$$
\gamma(a, \omega)=a^{-1} \omega^{a}{ }_{1} \digamma_{1}\left[\begin{array}{c|c}
a & \omega \\
a+1 & \omega
\end{array}\right] \quad(\Re\{a\}>0) .
$$

Finally, applying now the limiting process $\omega \rightarrow \infty$ to (14) and using (3), we deduce

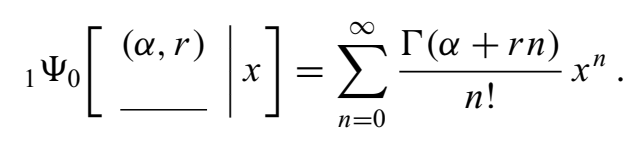

The gamma-, incomplete gamma- and the confluent hypergeometric ${ }_{1} \digamma_{1}$ are implemented in Mathematica as the subroutines Gamma $[z]$, Gamma $[a, 0, z]$ and HypergeometriclF1 [a,b, z], respectively.

B. In practice, the parameter vector $\theta=(\alpha, \mu, a, r)$ is unknown, and it has to be estimated from a random sample $\mathbf{X}=\left(X_{1}, \cdots, X_{n}\right)$, such that it consists of independently sampled identical random repliacæ of the r.v. $\xi \sim g W(\theta)$. The traditional parameter estimation procedure is the Maximum Likelihood (ML) method because the ML estimator possesses numerous desired properties, such as asymptotic normality and consistency. Unfortunately, this procedure can be used in a special case only for $r=1$, when $g W(\theta)$ becomes the ordinary gamma-distribution with parameters $\alpha$ and $\beta:=\mu+a$. It is known that the related ML-estimators are given by

$$
\ln \widehat{\alpha}-\psi(\widehat{\alpha})=\ln \frac{\bar{X}}{\sqrt[n]{\prod_{j=1}^{n} X_{j}}}, \quad \widehat{\beta}=\frac{\widehat{\alpha}}{\bar{X}}
$$

where $\psi=(\ln \Gamma)^{\prime}=\Gamma^{\prime} / \Gamma$ stands for the familiar digamma function, and $\bar{X}=\frac{1}{n} \sum_{j=1}^{n} X_{j}$ denotes the sample mean.

In the case when $r \neq 1$, in general the ML estimator does not exist. Indeed, let $0<r<1$. Then, the $\log$-likelihood function becomes

$$
\ell_{\theta}(\mathbf{X})=\ln \prod_{j=1}^{n} f_{g W}\left(X_{j}\right)=n\left(\alpha \ln \mu-\ln _{1} \Psi_{0}(\alpha, r)-(\alpha-1) \overline{\ln X}-\mu \bar{X}-a \overline{X^{r}}\right) .
$$

where

$$
{ }_{1} \Psi_{0}(\alpha, r)={ }_{1} \Psi_{0}\left[\begin{array}{c|c}
(\alpha, r) & \left.-\frac{a}{\mu^{r}}\right] \\
- & .
\end{array}\right.
$$


Equating to zero the partial derivatives of $\ell_{\theta}(\mathbf{X})$ with respect to $\mu$ and $a$, we conclude

$$
\begin{aligned}
{ }_{1} \Psi_{0}(\alpha, r) \mu^{r}(\alpha-\mu \bar{X}) & ={ }_{1} \Psi_{0}(\alpha+r, r) a r \\
{ }_{1} \Psi_{0}(\alpha+r, r) & =\mu^{r} \overline{X^{r}}{ }_{1} \Psi_{0}(\alpha, r)
\end{aligned}
$$

such that it results in

$$
\alpha-\mu \bar{X}=\operatorname{ar} \overline{X^{r}},
$$

when ${ }_{1} \Psi_{0}(\alpha, r) \cdot{ }_{1} \Psi_{0}(\alpha+r, r) \neq 0$. Now, it is not difficult to find values of $\theta$ for any fixed sample $\mathbf{X}$ outside of the manifold (15).

Similar procedure leads to counterexamples when $r>1$.

Since the ML method is not efficient when $r \neq 1$, following the recent method by Marković et al. (2009) developed for the three-parameter Weibull distribution, Least Squares parameter estimation method could be derived for the gamma-Weibull distribution. However, this problem deserves a separate article.

\section{ACKNOWLEDGMENTS}

The authors are thankful to the referees for giving useful suggestions in the improvement of this article. The present investigation was partially supported by the Ministry of Sciences, Education and Sports of Croatia under Research Project No. 112-2352818-2814.

\section{RESUMO}

A distribuição gamma-Weibull a cinco parâmetros foi introduzida for Leipnik e Pearce (2004). Nadarajah e Kotz (2007) a simplificaram para uma forma a quatro parâmetros usando funções hipergeométricas em alguns casos especiais. Nós mostramos que a função de distribuição cumulativa, todos os momentos de ordem positiva e a função característica da distribuição gamma-Weibull de uma variável randômica pode ser explicitamente expressa em termos da função-Psi de Fox-Wright confluente incompleta, recentemente introduzida por Srivastava and Pogány (2007). Ao mesmo tempo, generalizamos certos resultados de Nadarajah e Kotz que decorrem como casos especiais de nossos achados.

Palavras-chave: distribuição gamma, distribuição de Weibull, função ${ }_{1} \Psi_{0}$ de Fox-Wright confluente, função ${ }_{1} \psi_{0}$ de Fox-Wright confluente incompleta.

\section{REFERENCES}

GRADSHTEYN IS AND RYZHIK IM. 2000. Table of Integrals, Series, and Products. (Corrected and Enlarged Edition prepared by A. Jeffrey and D. Zwillinger), $6^{\text {th }}$ ed., Academic Press, New York.

LEIPNIK RB AND PEARCE CEM. 2004. Independent non-identical five-parameter gamma-Weibull variates and their sums. ANZIAM J 46: 265-271.

MARKović D, JukiĆ D AND Benšić M. 2009. Nonlinear weighted leat squares estimation of a three-parameter Weibull density with a nonparametric start. J Comput Appl Math 228: 302-312.

Mathai AM And SAXENA RK. 1978. The $H$-function with applications in statistics and other disciplines. Halsted Press, J Wiley \& Sons, New York-London-Sidney. 
NAdARajah S And Kotz S. 2007. On a distribution of Leipnik and Pearce. ANZIAM J 48: 405-407.

SRIVASTAVA HM AND PogÁNY TK. 2007. Inequalities for a unified family of Voigt functions in several variables. Russ J Math Phys 14: 194-200.

SRIVAstava HM, GuPTA KC And Goyal SP. 1982. The $H$-Functions of One and Two Variables with Applications. South Asian Publishers, New Delhi. 